Volume 9, No.1.4, 2020

International Journal of Advanced Trends in Computer Science and Engineering

Available Online at http://www.warse.org/IJATCSE/static/pdf/file/ijatcse1191.42020.pdf

https://doi.org/10.30534/ijatcse/2020/1191.42020

\title{
Performance Evaluation of Scheduling Schemes for VoIP Application in Device to Device (D2D) Communication
}

\author{
Yusmardiah Yusuf ${ }^{1}$, Darmawaty Mohd. Ali $^{2}$, Roslina Mohamad ${ }^{3}$ \\ ${ }^{1}$ Wireless Communication Technology, Fakulti Kejuruteraan Elektrik UiTM, ${ }^{1}$ ymardiahyusuf@ gmail.com \\ ${ }^{2}$ Wireless Communication Technology, Fakulti Kejuruteraan Elektrik UiTM, ${ }^{2}$ darma504@uitm.edu.my \\ ${ }^{3}$ Wireless High Speed Network, Fakulti Kejuruteraan Elektrik UiTM, ${ }^{3}$ roslina780@uitm.edu.my
}

\begin{abstract}
In recent years, there has been an increasing amount of research on Device to Device (D2D) communication underlying cellular network. D2D is a technology evolved from the next generation cellular network. In D2D communication, devices communicate with each other using a direct link without going through the evolved Node B (eNB). Nevertheless, there are still some challenges that have not been addressed by previous researchers. Most of the available studies are based on numerical or self-developed simulators. However, numerical or self-developed simulators do not resemble the real network and the real performance of the network cannot be gauged. This paper investigates the effect of scheduling algorithms in D2D communication for voice over internet protocol (VoIP) application using discrete even simulator, OMNET++. The study is performed using a single cell D2D communication environment using SimuLTE framework. The analysis is made by comparing several scheduling algorithms which are Deficit Round Robin (DRR), Proportional Fairness (PF) and Maximum Carrier to Interference (Max C/I). The number of D2D flows are varied from 5 to 30 and the results were analyzed in terms of throughput, delay, jitter and Mean Opinion Score (MOS). Simulation results show that Max C/I algorithm has a better quality of experience (QoE) which is proved by MOS value of 3.015. In terms of delay, the average VoIP packet scheduled by PF scheme is delayed by $0.33 \mathrm{~ms}$ compared to MAX C/I. The findings reported that Max $\mathrm{C} / \mathrm{I}$ gives a preferable performance in terms of throughput, jitter, delay and MOS value followed by PF and DRR.
\end{abstract}

Key words : Device to Device (D2D), scheduling algorithms, Long Term Evolution (LTE), OMNET++.

\section{INTRODUCTION}

In the past decades, mobile communication systems offer data, voice and video services only. In the history of development advanced communication devices, new services such as online gaming, video streaming and web browsing are supported by the network. These services usually have different requirement for bandwidth, delay and quality of service (QoS). Device to Device (D2D) technology has been studied by various researchers and has become one of the current discussion for a new generation of cellular networks. In relation to the D2D technology, new features and functionalities is introduced by $3 \mathrm{GPP}$ and need to be exploited into traditional cellular networks. Hence, the new D2D based cellular network or Long Term Evolution-Advanced (LTE-A) technology was introduced in [1].

Having the same element as an existing cellular LTE network, the LTE-A architecture that support D2D is consist of the evolved packet core (EPC) and the evolved universal terrestrial access network (E-UTRAN). The new architecture enhancement for D2D proximity services are described in [2]. The extended version of [2] are used as the specification of Third Generation Partnership Project (3GPP) standard to produce new version of release 12 that focused on public safety application requirements which provides communications for services such as police, fire and ambulances [3]. On the network side, the new capabilities was presented which are proximity-based service (ProSe) function and ProSe application server, and one new entity on the user side, ProSe application. ProSe communication has to work in regions, where network coverage cannot be guaranteed. Thus, three scenarios for D2D communication are considered which are within network coverage, out-of-coverage and partial coverage. D2D technology has shown noteworthy advantages to the current network such as higher resource allocation efficiency and peer-to-peer services [4].

One of the major problems in resource allocation is managing the limited available resources with varying conditions of the wireless channel to provision the QoS requirements. Different methods have been proposed to overcome this challenging problem and improve the overall performance of D2D communication. Scheduler plays a very important role in managing resource allocation. The scheduling operation is performed by a packet scheduler in the MAC layer [5]. The 
main function of the scheduler is to ensure efficient resource allocation being done among the UE in the network.

Therefore, the main goal of this paper is to evaluate the performance of deficit round robin (DRR), maximum carrier to interference $(\operatorname{Max} \mathrm{C} / \mathrm{I})$ and proportional fairness $(\mathrm{PF})$ schemes for voice over internet protocol (VoIP) application in D2D communication. The performance evaluation is conducted as the initial work for a new resource allocation algorithm to be deployed for D2D transmission The performance metrics of throughput, delay, jitter and MOS are analysed as it will affect the performance of the network. SimuLTE for OMNET++ [6] is applied in the evaluation.

The remainder of the paper is described as follows; Section 2 describes the related research works in D2D communication and scheduling algorithms in Section 3 respectively. Section 4 explains on the SimuLTE [6] framework and Section 5 describes QoS performance metrics. Whereas, section 6 presents the simulation configuration. Section 7 discusses the results and lastly, Section 8 draws the conclusion.

\section{REVIEW ON (D2D) COMMUNICATION}

T. Liu et. al [7] examined the effect of users' mobility on the resource allocation for general multi-hop D2D communication underlaying cellular networks. To increase the user throughput, the researchers proposed an efficient square-division-based resource allocation algorithm which was adopted by square tessellation technique. At the same time, the resource allocation, users' mobility and relay-based framework was designed as a relay-assisted D2D communication. Simulation results revealed that, the relay-assisted for D2D communication protocol improved the system throughput up to $55 \%$ and the user access rate up to four times.

X. Zhang and J. Wang [8] had investigated on the heterogeneous statistical quality of service (QoS)-driven resource allocation method. The Nakagami-m fading model was established for the dynamic D2D cluster-caching system purposely for video stream application. The heterogeneous statistical QoS constraints was derived based on the 5G multimedia mobile wireless network. A simulation was conducted to analyze and explore the proposed D2D cluster-caching based algorithm in terms of effective capacity under heterogeneous statistical QoS limitation. The proposed scheme achieved better performance under heterogeneous statistical delay-bounded QoS constraints.

Based on the proportional fairness (PF) criteria, a fair scheduling for D2D communication in an OFDMA-based cellular network was presented in [9]. A PF-based scheduling algorithm was proposed to increase the sum of acceptable rate for D2D and cellular users. This scheme was called hybrid optimization problem since the power control allocation and spectrum sharing were included. Based on the transmit power limitation and QoS requirement, firstly, the allowable region was decided. After that, the joint transmit power for the cellular user and D2D pair in the allowable area was calculated. Authors also proved that the ideal value was sorted-out from several points on the borderline of the permitted area. Finally, multiple sub-channels were occupied for scheduling scheme that was based on the Hungarian algorithm and generated bipartite graph. In order to achieve maximal matching, the reusable cellular users and D2D pairs were selected to increase the sum of the achievable data rates. Authors claimed that PF-based algorithm can guarantee the excellent performance of fairness and throughput in D2D communication.

In the literature review of [7-9], it is found that the authors used complex mathematical solutions based on numerical analysis [7-8] and self-developed simulators [9] to design the schemes for resource allocation which are not applicable for implementation in real environment. These techniques do not resemble the real network and the real performance of the network cannot be determined.

Asheralieva et.al [10] had implemented their proposed algorithms using a system level simulators called OPNET. The researchers adapted the QoS in their proposed resource allocation algorithm by minimizing the interference to stay within a predefined threshold. However, the efficiency of the proposed algorithm was evaluated for the QoS parameters of delay and packet loss rate and exclude the performance of throughput which is a very important parameter to satisfy the QoS.

Another studies made by K. Dhivya and C. Arun [11] using OMNET++. They proposed the algorithm named Iterative Resource Allocation and Power Control scheme (IRAPC). The study was set up to increase D2D users' sum rate. The D2D user select the mode of transmission based on the distance between transmitting D2D user and receiving D2D users. The algorithm proposed by the authors consider the link quality of D2D user and the interference that exits in the network. The performance of the network can be optimized by increasing the received signal-to-interference-and-noise ratio (SINR) or sum-rate. However, no data regarding the QoS performance evaluation of D2D communication is discussed.

A. Virdis, G. Nardini, and G. Stea [12] has introduced the framework called SimuLTE. They presented a model for unicast D2D communication underlying cellular network. The authors explain on how to model one-to-one D2D communications from a system-level point of view and the 
procedure to integrate the model into the SimuLTE simulator was presented. To conclude this section, the literature identifies that only the works in [10-12] have implemented their proposed algorithms for D2D communication using the system level simulators which is OPNET, OMNET++and SimuLTE.

\section{SCHEDULING ALGORITHMS}

The packet scheduler allocates and manages packets according to the service types. It is located in the media access control (MAC) layer. Packet scheduling schemes is one of the functions in radio resource management (RRM). It is in control for packets transmission and user selections to satisfy the radio resources utilizations and QoS requirements [1]. When load of packets are waiting to be served, the scheduling algorithm served the packets according to priority metrics to guarantee their QoS requirement. Deficit round robin (DRR), Proportional fairness (PF) and Maximum carrier to interference (Max $\mathrm{C} / \mathrm{I})$ are the types of schedulers to be discussed in this section.

\subsection{Maximum Carrier to Interference Ratio (Max C/I)}

Maximum carrier to interference ratio (Max C/I) is one of the scheduling policies in D2D network [12]. Max C/I schedule the users in the network based on the Channel Quality Indicator (CQI) value and allocate the Resource Blocks (RBs) to the users.

\subsection{Proportional Fairness (PF)}

Proportional fair (PF) algorithm was designed to deal with the problems of user fairness and throughput. The PF scheme provides a balance between them [13]. For a given number of time slots, the algorithm assigns user based on average throughput among the active users in the system.

\subsection{Deficit Round Robin (DRR)}

Deficit Round Robin (DRR) is the basic scheduling scheme. The RR algorithm allocates equal portions of packet transmission time to each user. It is on a first come first served basis [14]. Either users have different channel conditions or sized packet waiting at their buffers, still they will be allocated in the fairly manner.

\section{SIMULTE FRAMEWORK}

SimuLTE framework was created by group of researchers to examine the complex network environments. It is based on the OMNeT++ [15] framework. Each module is defined through NED files, and a behavior, implemented via $\mathrm{C}++$ classes. A high-level representation contains the LTE (network interface card) NIC, together with modules implementing upper layer protocols, taken from INET library is to implement entities outside the LTE scope, such as application servers [6]. In addition, the eNB has an interface to the Internet via PPP and can also be connected to other eNBs using the X2 interface The LTE NIC in both the UE and the eNB implements the whole LTE protocol stack, as one submodule per layer, namely packet data convergence protocol (PDCP), radio link control (RLC), MAC and physical (PHY). Each layer includes common and node-specific functionalities. Communication between different layers occurs via message exchange, same as data transmission between UEs and eNBs.

A dedicated module, called Binder, monitors which resource blocks (RBs) are used by both the eNBs (for downlink transmissions) and the UEs (for uplink transmissions). The binder can be considered as the oracle of the LTE network, since all the LTE nodes can access it to share common information via direct method calls. SimuLTE provides models of real-life applications such as VoIP. The internet protocol (IP) module is taken from the INET package. In the UE side, the IP connects the NIC to applications that use transmission control protocol (TCP) or user datagram protocol (UDP). Whereas in the eNB, the IP connects the eNB to other IP peers for example a web server, via point-to-point protocol (PPP) [6].

\section{QOS PERFORMANCE METRICS}

The performance of scheduling schemes for VoIP application were based on the following parameters which are throughput, jitter, MOS and delay.

\subsection{Throughput}

Throughput is measured as the data transfer rate when delivering packets to all the terminal in the network [16]. It indicates the network's performance and quality when delivering packets from one point to another. The unit is bits per second (bps).

\subsection{Delay}

Delay can be defined as a period for transmitting packets from source to the destination. It is important to measure the delay as it contributes to the Quality of Experience (QoE) that measure the satisfaction of user towards the network. The value of delay must be less than $150 \mathrm{~ms}$ to ensure excellent performance as recommended by ITU [17]. Table 1 shows the recommended values for packet loss, delay, and jitter as described by ITU from poor to excellent quality of performance.

Table 1: ITU Recommended Values For VoIP Quality [17]

\begin{tabular}{|l|l|l|l|}
\hline Performance & Delay & Jitter & $\begin{array}{l}\text { Packet } \\
\text { loss }\end{array}$ \\
\hline Excellent & $<150 \mathrm{~ms}$ & $<20 \mathrm{~ms}$ & $<1 \%$ \\
\hline Good & $>150 \mathrm{~ms}<$ & $>20 \mathrm{~ms}<50$ & $>1 \%$ \\
& $300 \mathrm{~ms}$ & $\mathrm{~ms}$ & $<5 \%$ \\
\hline Poor & $>300 \mathrm{~ms}$ & $>50 \mathrm{~ms}$ & $>5 \%$ \\
\hline
\end{tabular}




\subsection{Jitter}

Jitter is defined as the average of the absolute difference in the time taken for successive packets to reach the destination [17]. Jitter determine the network performance and it is used as the stability and consistency indicator of a network. It assures the Quality of Experience (QoE) where the value must be less than $50 \mathrm{~ms}$ according to the QoS parameter from 3GPP.

\subsection{Mean Opinion Score}

MOS is the overall quality of D2D link network where it takes all the conditions that disturb the network quality into consideration. Voice quality is typically expressed in terms of the MOS, which its subjective rating of audio quality on a scale from 1 to 5 [18]. The MOS value reflects the user experience. Table 2 tabulates the MOS score for for VoIP transmission.

Table 2: MOS Quality Indicator [18]

\begin{tabular}{|c|c|}
\hline MOS & Quality \\
\hline 5 & Excellent \\
\hline 4 & Good \\
\hline 3 & Fair \\
\hline 2 & Poor \\
\hline 1 & Bad \\
\hline
\end{tabular}

\section{SIMULTE ENVIRONMENT AND SIMULATION CONFIGURATION}

This section presents the OMNET++ LTE network architecture which is developed by using SimuLTE [6] and the parameters used in the simulation. A single cell D2D network is considered. The system model in Figure 1 describes the configuration of a scenario where two UEs are in the modes of transmitting the packets using D2D communications. It is composed of one eNB, one user equipment (UE) transmitting using D2D called ueD2DTx, and one UE receiving using D2D called ueD2DRx.

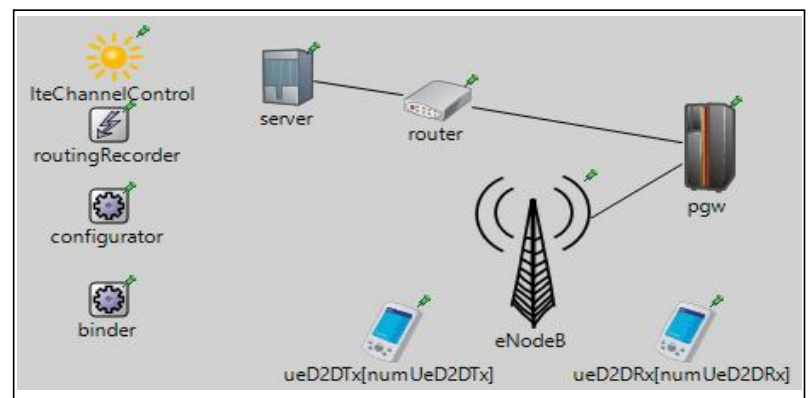

Figure 1: Single Cell Scenario for D2D UE in SimuLTE

The other elements in the model is an access gateway (PGW) which provides the network with the data flow control besides to help the network with the flow control. Lastly, one server which is a standard host to provide signaling services. The server is linked to the PGW of the LTE network with a speed link of 10 gigabits per second (Gb/s). Each D2D UEs run a VoIP application using user datagram protocol (UDP) as transport layer protocol. Each flow of traffic is generated by a server and passes through the gateway to the eNB. The eNB maintains a queue for each traffic where it will wait for the transmission to the associated UE.

The D2D UEs are $50 \mathrm{~m}$ away from the eNB and the distance of D2D UE is fixed to $20 \mathrm{~m}$ from each other to ensure successful transmission [11]. If the D2D UEs' distance is more than $20 \mathrm{~m}$, the user will be directed to the traditional cellular communication mode. The number of UEs are configured using the corresponding NED parameters and D2D flows are distributed randomly in a single cell scenario under network controlled environment. The number of D2D UEs inside the single cell scenario varies from 5 to 30 and the UEs are transmitting using D2D mode only.

Voice over internet protocol (VoIP) application is used as an input traffic. VoIP delivers D2D UEs conversation through the internet as packets of data. In order to send voice as data, the sound is converted into packets. The ueD2DTx is sending one packet every $20 \mathrm{~ms}$. Packets are sent in intervals to maintain the flow of conversation. The packet size is fixed to 40 bytes and the performance of the D2D flow is assessed with different number of users. The simulation parameters are provided in Table 3 and simulation scenario is depicted in Figure 2 respectively.

Table 3: Simulation Parameters

\begin{tabular}{|l|l|}
\hline Parameter & Value \\
\hline Scheduler & DRR, PF, Max C/I \\
\hline Carrier frequency & $2 \mathrm{GHz}$ \\
\hline Bandwidth & $10 \mathrm{MHz}$ \\
\hline Mobility model & Static \\
\hline Path loss model & ITU Urban Micro \\
\hline Fading model & Jakes \\
\hline Simulation time & $10 \mathrm{~s}$ \\
\hline UE Tx power & $26 \mathrm{~dB}$ \\
\hline D2D UE pairs & 30 \\
\hline
\end{tabular}

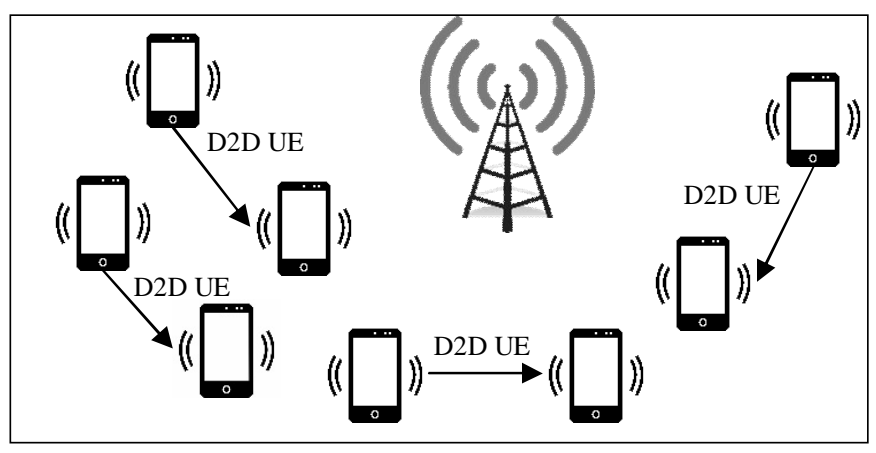

Figure 2: Simulation Scenario

A scheduling schemes allocates radio resources to D2D users by a specific priority metric for example Max C/I choose the best channel quality to be schedule first. The resource 
allocation among users is based on limited of physical resource blocks $(\mathrm{PRB})$ provided. In this simulation, in order to demonstrate the performance of scheduling algorithms, the simulation for Max C/I, PF and DRR is conducted. The performances of scheduling algorithms for VoIP application are evaluated using OMNeT++ version 5.1 simulator. The performance comparison in terms of throughput, jitter, delay and MOS score are recorded accordingly.

\section{PERFORMANCE EVALUATION}

This section provides the results for the conducted experiment and is analyzed in terms of throughput, delay, jitter and MOS score.

\subsection{VoIP Throughput versus D2D UEs}

VoIP received throughput mean is the average value of throughput achieved by all D2D users. The average values of D2D user throughput using Max C/I, PF and DRR algorithm are illustrated in Figure 3.

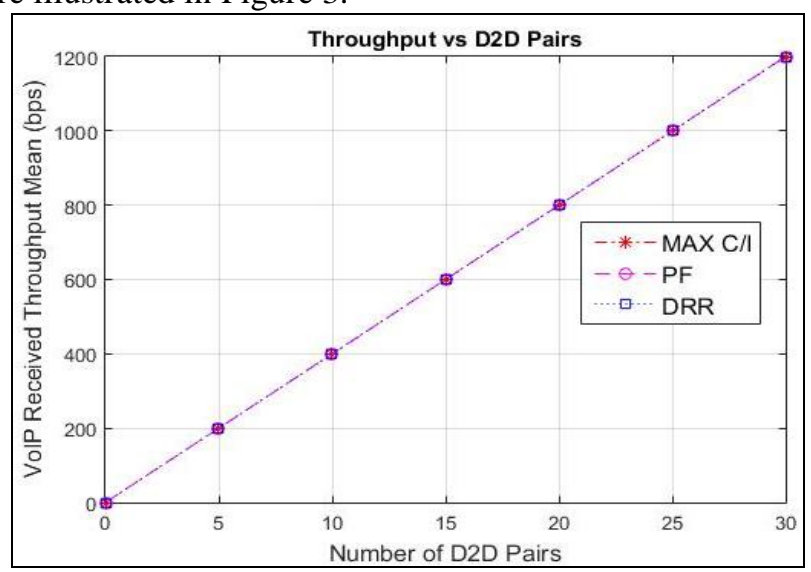

Figure 3: Average VoIP Received Throughput

In Figure 3, the number of D2D users is varied from 5 to 30. The average D2D user throughput value increased as the number of D2D UEs increased for all algorithms because the VoIP packet are successfully received by ueD2DRx.

\subsection{Delay Versus D2D UEs}

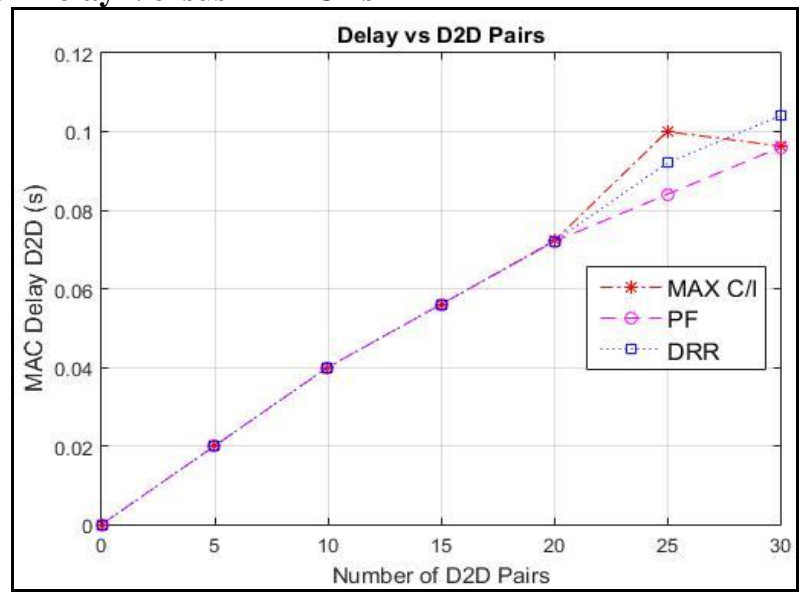

Figure 4: Average Delay
Figure 4 shows the delay of the D2D UEs when the transmitting UEs are delivering VoIP application to the receiving UEs. The delay value increases for all the algorithms when the number of UE pairs increases. It is mainly due to the traffic routing and interference between D2D users in the network when the devices are trying to connect to each other. The average delay for Max C/I to transmit all the packets is lesser than PF and DRR. This is due to the successful pairing between the UEs which means the voice transmission is completed. RR and PF schedulers show similar trending where the delay increased as the user increased. However, PF shows the lowest delay variation compared to RR. Based on ITU standard, the QoE for VoIP user is achieved when the delay is below $150 \mathrm{~ms}$. Furthermore, the delay performance for Max C/I, PF and DRR algorithms are still provisioning the QoS when the UEs are increased up to 30 D2D UE pairs.

\subsection{Jitter Versus D2D UEs}

Jitter occurs when packets of information are dropped, or sent out of order. Based on ITU standard, the jitter value must be less than $50 \mathrm{~ms}$ to ensure the user QoE to guarantee the network performances.

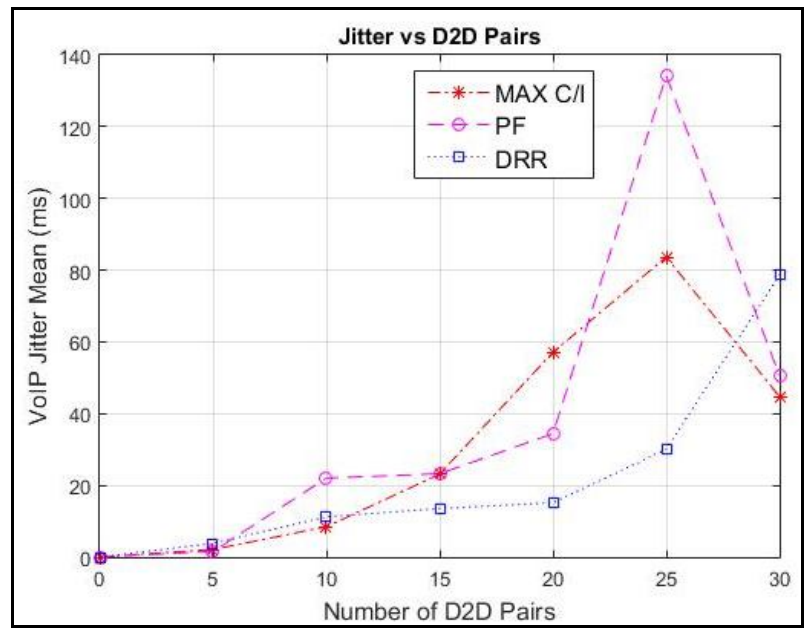

Figure 5: Average Jitter

Figure 5 shows the average VoIP jitter for the D2D UEs. According to the standard, if the jitter is exceeding $50 \mathrm{~ms}$ delay, the call quality will reduce tremendously. From Fig. 7, Max C/I have the lowest average jitter value when delivering the VoIP packet. For DRR algorithm, as the user increased, the delay also increases because congestion occurs in the network. DRR schedules users without any priority hence lead to insufficient bandwidth to manage the VoIP call. In return, the packets were delivered out of order. Meanwhile, PF algorithm has the highest jitter value when UE is increased to 25. This algorithm selects flows having maximum data rate causing the jitter value escalated. Moreover, the Max C/I shown better value when the user is increased up to $30 \mathrm{D} 2 \mathrm{D}$ pairs. This is due to good channel quality of D2D flows which results in lesser delays when transmitting voice packet to the ueD2DRx. 


\subsection{MOS Score}

Figure 6 shows the MOS value for D2D UEs. An excellent MOS value is equal to 5 . Max C/I algorithm gives the lowest MOS value when the number of D2D pairs is 20 . This is due to bad channel quality experienced by the D2D UE at that time hence Max C/I having the lowest value. Overall MOS score for Max C/I, PF and DRR gives score of 2.8 to 3 for voice quality of the network which is fair.

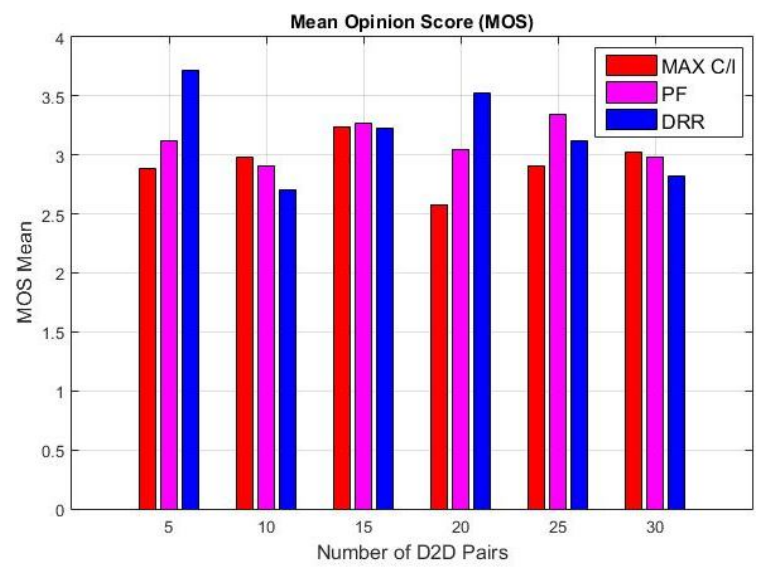

Figure 6: MOS Score

Table 4 summarized the performance of Max C/I, PF and DRR for 30 D2D flows. The Max C/I shows a preferable performance of throughput, jitter, delay and MOS as the D2D UEs increased followed by PF and DRR. It can be concluded that, under increasing D2D traffic, the performance of delay and jitter in D2D communication is depending on the selection of scheduling algorithm. The delay and jitter are also affected by network configuration and geographical size.

Table 4: Performance Evaluation Summary

\begin{tabular}{|c|c|c|c|}
\hline QoS Parameter & MAX C/I & PF & DRR \\
\hline Throughput (bps) & 1200 & 1200 & 1200 \\
\hline Jitter (ms) & 44.74 & 50.55 & 78.69 \\
\hline Delay (ms) & 96.33 & 96.00 & 104.00 \\
\hline MOS & 3.015 & 2.978 & 2.817 \\
\hline
\end{tabular}

\section{CONCLUSION}

This paper has initiated the idea of determining the suitable scheduling algorithm for D2D communication whilst provisioning the QoS. This paper has investigated the performance of Max C/I, PF and DRR algorithm for VoIP traffic in D2D communication in terms of throughput, delay, jitter and MOS value. It can be concluded that the Max C/I shows a preferable performance of throughput, jitter, delay and MOS as the D2D UEs increased followed by PF and DRR.

For future recommendation, the performance of different peer-to-peer application such as video streaming or file transfer can be investigated for the D2D communication. This study focused on scheduling algorithm only for D2D communication. Hence, the performance of D2D underlaying the cellular network should also be analysed. Furthermore, a new scheduling algorithm and mode selection algorithm should be developed so that the efficiency of D2D communication underlying cellular network can be improved mainly in terms of interference.

\section{ACKNOWLEDGEMENT}

This work was supported by the Ministry of Higher Education under FRGS Grant, Ministry of Higher Education Malaysia 600-IRMI/FRGS 5/3 (047/2019). We would like to extend our acknowledgment to Fakulti Kejuruteraan Elektrik Universiti Teknologi Mara (UiTM) Shah Alam and those who have directly or indirectly contribute to this research.

\section{REFERENCES}

1. 3GPP. LTE Network architecture, in 3GPP TS 23.002 version 12.6.0 Release 12, 2015.

2. G. T. 23.703. Study on architecture enhancements to support proximity services (ProSe)," in Technical Specification Group Services and System Aspects, 2013.

3. 3GPP. Proximity-based services (ProSe); Release 12) in "3GPP TS 23.303 version 12.2.0 Release 12), 2014.

4. J. Liu, N. Kato, J. Ma, and N. Kadowaki. Device-to-Device Communication in LTE-Advanced Networks: A Survey, in IEEE Communications Surveys \& Tutorials, vol. 17, no. 4, pp. 1923-1940, 2015.

5. S. Balakrishnan, A. Sivasubramanian, and S. P. K. A review of MAC scheduling algorithms in LTE system, in International Journal on Advanced Science, Engineering and Information Technology, vol. 7, 2017.

6. A. Virdis, G. Nardini, and G. Stea. Cellular-Networks Simulation Using SimuLTE, in Recent Advances in Network Simulation (EAI/Springer Innovations in Communication and Computing, 2019.

7. T. Liu, J. C. S. Lui, X. Ma, and H. Jiang. Enabling Relay-Assisted D2D Communication for Cellular Networks: Algorithm and Protocols, IEEE Internet of Things Journal, vol. 5, no. 4, pp. 3136-3150, 2018.

8. X. Zhang and J. Wang. Heterogeneous Statistical QoS-Driven Resource Allocation for D2D Cluster-Caching Based 5G Multimedia Mobile Wireless Networks, 2018.

9. W. Zheng, J. Hu, C. Liu, and Y. Fu. Cellular-D2D resource reuse algorithms based on proportional fairness, in EURASIP Journal on Wireless Communications and Networking, vol. 2017, no. 1, 2017. https://doi.org/10.1186/s13638-017-0953-9

10. A. Asheralieva and Y. Miyanaga. QoS-Oriented Mode, Spectrum, and Power Allocation for D2D 
Communication Underlaying LTE-A Network, in IEEE Transactions on Vehicular Technology, vol. 65, no. 12, pp. 9787-9800, 2016.

11. K. Dhivya and C. Arun. Establishing Dvice to Device Communication Under Cellular Architecture, presented at the Conference on Emerging Devices and Smart Systems (ICEDSS 2018), India, 2018.

12. A. Virdis, G. Nardini, and G. Stea. Modeling unicast device-to-device communications with SimuLTE, in 2016 1st International Workshop on Link- and System Level Simulations (IWSLS), 2016.

13. N. Ferdosian and M. Othman. Two-Level QoS-Oriented Downlink Packet Schedulers in LTE Networks: A Review, in Proceedings of the First International Conference on Advanced Data and Information Engineering (DaEng-2013) (Lecture Notes in Electrical Engineering, 2014.

14. QoS Scheduling Algorithm with Improved MAX C/I for the Performance Enhancement of WiMAX Enviornment, 2016.

15. M. M. Shafiei and A. J. Jameel. Performance Analysis of Voice Over LTE Using OMNeT++, in, Journal of Electrical \& Electronic Systems, Vol. 6, Issue 12017.

16. S. K. Talha and B. I. A. Barry. Evaluating the Impact of AES Encryption Algorithm on Voice over Internet Protocol (VoIP) Systems, in 2013 International Conference On Computing, Electrical and Electronic Engineering (ICCEEE), Khartoum, Sudan, 2013.

17. B. Kumar and P. Gupta. Scheduling Algorithms in a WiMAX Network," in 2nd International Conference on Advanced Computing \& Communication Technologies, 2012.

18. A. Vizzarri and F. Davide. Quality of Experience Analysis for VoLTE Services through Artificial Neural Network Fitting, 2016. https://doi.org/10.1109/WINCOM.2016.7777208 